\title{
Comparison of a Segmentation Algorithm to Six Expert Imagiologists in Detecting Pulmonary Contours on X-ray CT Images
}

\author{
Carlos Ferreira $^{a, b}$, Beatriz Sousa Santos ${ }^{c, d}$, Jose Silvestre Silva ${ }^{c, e}$, Augusto Silva ${ }^{, c, d}$ \\ ${ }^{a}$ Departamento de Economia, Gestão e Engenharia Industrial, Universidade de Aveiro, Portugal \\ ${ }^{b}$ Centro de Investigação Operacional, Faculdade de Ciências, Universidade Lisboa, Portugal \\ ${ }^{c}$ Departamento de Electrónica e Telecomunicações, Universidade de Aveiro, Portugal \\ ${ }^{d}$ Instituto de Engenharia Electrónica e Telemática de Aveiro, Universidade de Aveiro, Portugal \\ ${ }^{e}$ Departamento de Física, Universidade de Coimbra, Portugal
}

\begin{abstract}
Quantitative evaluation of the performance of segmentation algorithms on medical images is crucial before their clinical use can be considered. We have quantitatively compared the contours obtained by a pulmonary segmentation algorithm to contours manually-drawn by six expert imaiologists on the same set of images, since the ground truth is unknown.

Two types of variability (inter-observer and intra-observer) should be taken into account in the performance evaluation of segmentation algorithms and several methods to do it have been proposed. This paper describes the quantitative evaluation of the performance of our segmentation algorithm using several figures of merit, exploratory and multivariate data analysis and non parametric tests, based on the assessment of the inter-observer variability of six expert imagiologists from three different hospitals and the intra-observer variability of two expert imagiologists from the same hospital.

As an overall result of this comparison we were able to claim that the consistency and accuracy of our pulmonary segmentation algorithm is adequate for most of the quantitative requirements mentioned by the imagiologists. We also believe that the methodology used to evaluate the performance of our algorithm is general enough to be applicable to many other segmentation problems on medical images.
\end{abstract}

Keywords: Quantitative Evaluation, Computed Tomography, Inter- and Intra-Observer Variability, Pulmonary Contours, Multivariate Data Analysis, Non-Parametric Statistics

\section{INTRODUCTION}

Contour oriented segmentation often occurs as a pre-processing step of a more global image analysis task, as it is the case of computer-aided analysis of pulmonary X-ray tomograms. Most algorithms for segmentation of pulmonary regions are based on intensity discrimination within the Hounsfield scale, however this task may be very complex ${ }^{1-3}$. In a previous work ${ }^{4}$ we have presented algorithms that generate contours with a variable degree of similarity to those provided by expert imagiologists.

A quantitative evaluation of the performance of segmentation algorithms is crucial before their clinical use can be considered. Still, the performance evaluation of these algorithms in medical imaging is recognized as a difficult problem; actually, if one can find in the literature a significant number of contributions concerning the overall segmentation problem by itself, the same is not true when looking for quality and effectiveness assessments performed in some systematic way and having a practical value ${ }^{5}$.

This evaluation encounters as first great obstacle the fact that the ground truth is unknown; i.e. it is not possible to identify the real contour corresponding to a given image, we can only know contours manually-drawn by expert imagiologists on that image. Yet, not only contours drawn by two imagiologists will be different (inter-observer variability) but also there will not be agreement between contours drawn by the same imagiologist at different occasions 
(intra-observer variability). These two types of variability have to be taken into account in the performance evaluation of segmentation algorithms and several methods to do it have been proposed ${ }^{6-8}$.

We have chosen to compare quantitatively the contours obtained by our pulmonary region segmentation algorithm to the manually-drawn contours by expert imagiologists on the same set of images. We had already verified that a greater similarity existed between the contours produced by our algorithm and the contours drawn by two expert imagiologists, than between the contours drawn by the same two imagiologists ${ }^{9}$. This means that the inter-observer variability between our algorithm and any of the two imagiologists is smaller than the inter-observer variability between the two imagiologists. To investigate if this was specific for those two imagiologists, we have performed a study including six imagiologists. We have also considered relevant the study of the intra-observer variability; even if in this respect our algorithm has a clear advantage, as its intra-observer variability is zero, we expected this study to provide interesting information to the inter-observer study.

In this paper we describe the quantitative evaluation of the performance of our pulmonary segmentation algorithm using several figures of merit, Exploratory Data Analysis (EDA), Multivariate Data Analysis (MDA) and Non Parametric Tests. This evaluation was based on the assessment of intra-observer variability of two experts from the same imagiology department and the comparison to reference contours obtained from the contours manually-drawn by six expert imagiologists from three different departments. The use of reference contours that correspond to "mean" contours, seemed acceptable in most images with diagnosis value.

As an overall result of this comparison we were able to claim that the consistency and accuracy of our detection method is adequate for most of the quantitative requirements mentioned by the imagiologists.

The used methodology was developed specifically to quantitatively evaluate the performance of our pulmonary region segmentation algorithm; however we believe it is general enough to be applicable to many other problems of segmentation on medical images.

\section{METHODS}

Making accurate, unbiased comparisons of performance is in general a very difficult task, however some guidelines have been identified as relevant and helpful ${ }^{6,10}$. We have been using a set of guidelines that can be found in ${ }^{4}$, from which we stress the following ones: report results on common test datasets; use an adequate methodology to choose the test datasets; choose carefully the observers and methods used to assess the ground-truth; define the conditions in which the observers will operate; clearly specify the used performance metrics (figures of merit); choose hypothesis tests compatible with the figures of merit; use non-parametric tests if the statistical distribution of the data is unknown; use paired test if all the methods can be applied to the same image.

In this section we describe how we have compared the performance of our pulmonary segmentation algorithm to the performance (as pulmonary contour detectors) of six experts of different imagiology departments. In order to assess if the algorithm is an acceptable contour detector, we have adopted a twofold approach: on one hand we have studied the inter-observer variability among all contour detectors (imagiologists and algorithm) and, on the other hand, we have studied the intra-observer variability of two experts.

The study of the inter-observer variability was performed comparing each detected contour (by expert or algorithm) to a "mean" contour that, in the absence of ground truth, has been computed to be used as a reference. This study was expected to assess if our algorithm is comparable to the six experts as a pulmonary contour detector on the used test dataset.

The contours produced by our segmentation algorithm on an image are always the same; in this respect our algorithm has a clear advantage over the human experts since it has zero intra-observer variability, which is not the case of the humans. However, studying the intra-observer variability of two experts from the same imagiology department (but having a significantly different amount of experience) was expected to yield a rough estimate of the expected range of intra-observer variability. Moreover, these values were likely to add extra information to assess the significance of the inter-observer variability between the algorithm and any expert since they could be used as a relative measure of acceptable variability. 
We have used several figures of merit, Exploratory Data Analysis (EDA), Non Parametric Tests and Multivariate Data Analysis (MDA).

\subsection{TEST DATA SET}

We have used a test data set composed of 30 images (60 contours) randomly selected from 253 images, with diagnosis value, of 8 patients with various pathologies, not including images from traumatized patients that could have pulmonary regions of uncommon shapes.

\subsection{MANUALLY DRAWN CONTOURS}

To obtain digital versions of the manually drawn contours we have asked six expert radiologists from three different imagiology departments to manually-draw the contours on transparent sheets superimposed on hardcopies of 30 images. To register the curves as the right and left contours, the image Radon transforms for 0 and 90 degrees ${ }^{11}$ were calculated and from the maximum values of these two transforms we obtained an estimate of the centre of each lung. Applying a morphologic filling ${ }^{12}$ starting from the centre of one lung and a second filling starting from any point external to the lungs, we obtained an image containing the filled area of the other lung. The contour of the lung was then easily obtained. Erosion was applied to obtain a thinner version of each of the contours ${ }^{4}$.

\subsection{REFERENCE CONTOUR}

As we haven't access to the ground truth, i.e. the "real” pulmonary contour corresponding to each image, we have used reference contours obtained from the six contours detected by the expert imagiologists on each image. For most images having diagnosis value, the contours detected by all the imagiologists are only slightly different and thus using a "mean” contour as a reference contour seemed an acceptable approach (figure 1).

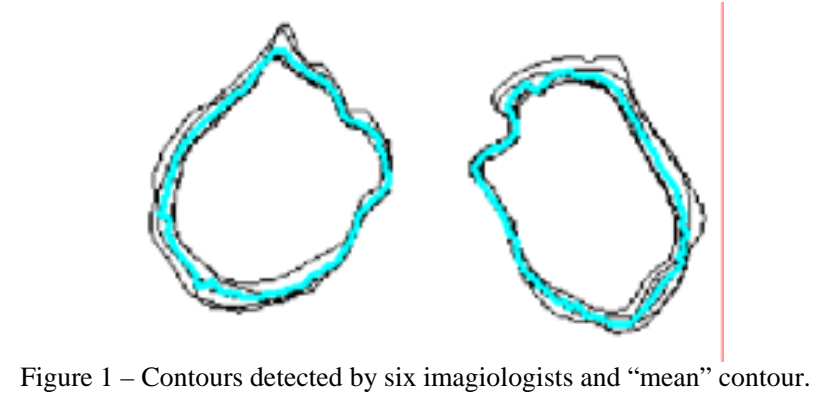

To obtain the "mean" contour corresponding to each image we have used the algorithm described below and illustrated in figures 2 through 7 for the set of three example geometric contours shown in figure 2. From $n$ contours (drawn by $n$ experts) this algorithm computes a reference contour having $i$ points and consists on the following steps:

1- fill in every contour with “1”, obtaining $n$ binary images (figure 3);

2- find the AND and the OR of all the images (figure 4);

3- find the XOR of the two images obtained in 2) (figure 5a);

4- find the skeleton of the shape obtained in 3) (figure 5b);

5- for each point $i$ of the skeleton obtained in 4) (figure 6):

5.1- find a perpendicular $\mathbf{r}$ to the approximate tangent line (computed using a neighbourhood of 20 points);

5.2- find the $n$ intersections of $\mathbf{r}$ to each given contour (e.g. obtaining points A, B and C or P, Q and T);

5.3- find the mean point $\mathrm{M}_{\mathrm{i}}$ of the $n$ intersection points obtained in 5.2);

6- the "mean" contour of the $n$ given contours is defined by $i$ points computed in 5.3) (figure 7). 

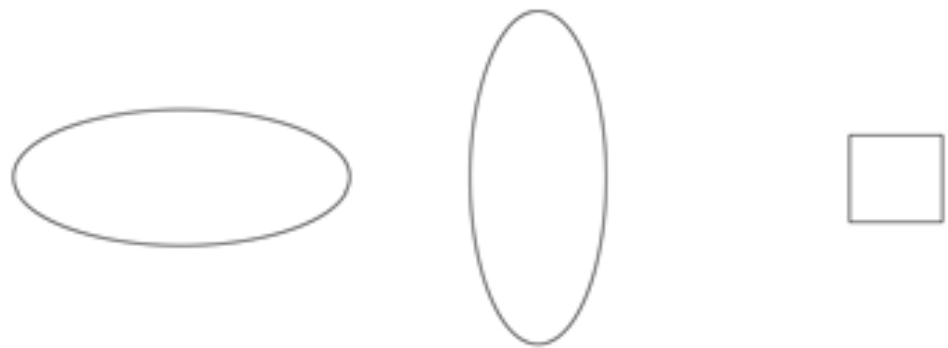

Figure 2- Three given contours.
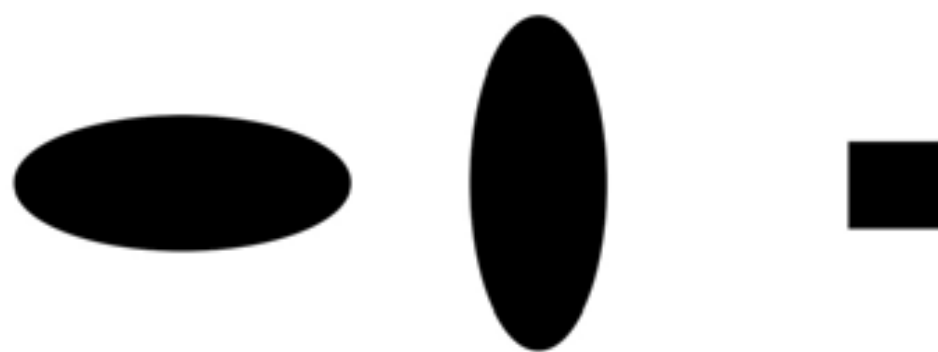

Figure 3- Binary images obtained by filling in the given contours.
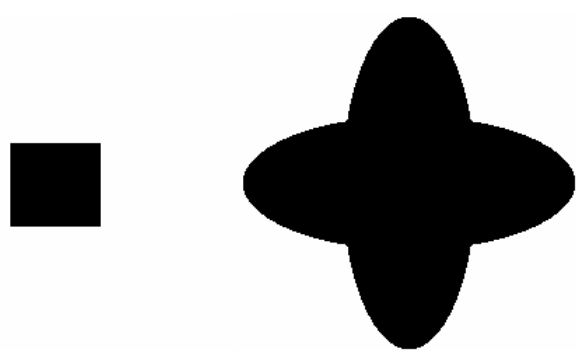

Figure 4- AND and OR of the three binary images.
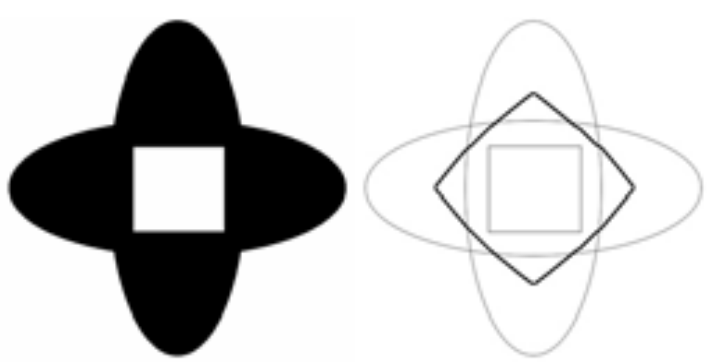

Figure 5- XOR (a) of the two binary images and skeleton (b).
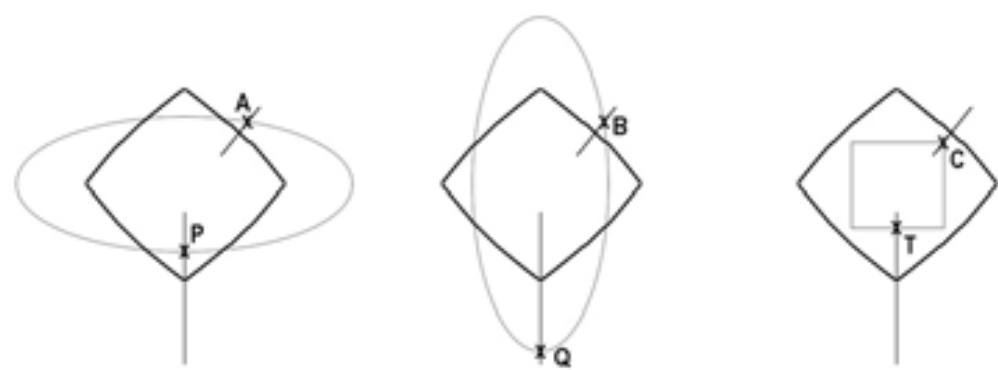

Figure 6- Points obtained from the three given contours. 


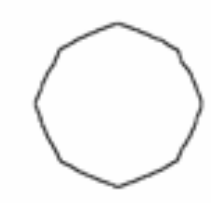

Figure 7- "Mean” contour of the three given contours.

\subsection{COMPARISON OF CONTOURS}

In order to compare any two contours using the distances between them, it is necessary to find pairs of corresponding points (one from each contour) and to determine distances between these points. Let $A$ and $B$ (figure 8) be two similar closed lines corresponding to the contour of a lung:

$$
\text { Contour } A=\{\text { set of } n \text { points }\} \quad \text { Contour } B=\{\text { set of } m \text { points }\}
$$

each point belonging to an image.

Let areas C, D and E be:

$$
\begin{gathered}
\mathrm{C}=\text { area enclosed by contour } A \quad \mathrm{D}=\text { area enclosed by contour } B \\
\mathrm{E}=\mathrm{C} \text { XOR D }
\end{gathered}
$$

$F$ is defined as a closed line existing inside area $E$ in zones where contour $A$ is not equal to $B$, coincident with these contours otherwise (figure 9).

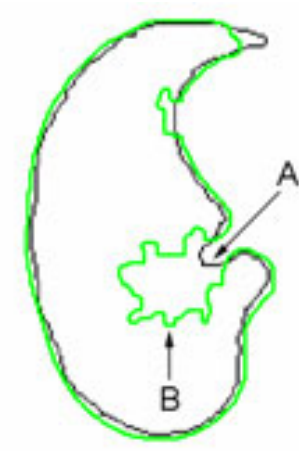

Figure 8 - Example of lung contours $A$ and $B$.

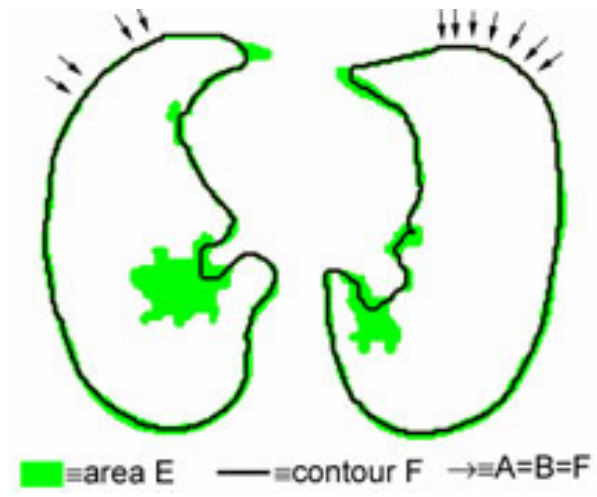

Figure 9 - Line $F$ (black) inside area $E$ (grey) and a zone where $F \equiv A \equiv B$.

Perpendicular lines $\dagger$ to contour $F$ are obtained for each point of this contour. The interception of each of these lines with contours A and B defines a pair of corresponding points on both contours (figure 10). Finally Euclidean distances are computed between all the corresponding points.

\footnotetext{
${ }^{\dagger}$ Perpendicular lines are computed as orthogonal to the tangents to each point of $F$ using neighbourhoods of 20 contour points. The size of this neighbourhood is a parameter that is related to the sensitivity to local characteristics of line $F$ and had to be adjusted.
} 


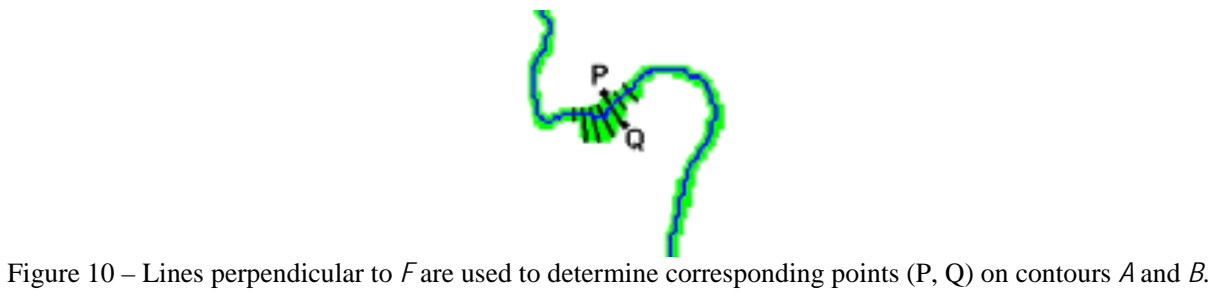

\subsection{FIGURES OF MERIT}

We have used several figures of merit ${ }^{4}$ to assess the similarity between any two contours:

- $\quad$ Pratt figure of merit,

- $\quad$ mean distance,

- maximum distance,

- $\quad$ similarity angle.

The Pratt figure of merit ${ }^{13}$ is defined as:

$$
F_{\text {Pratt }}=\frac{1}{N} \sum_{i=1}^{N} \frac{1}{1+\alpha \times d_{i}^{2}}
$$

where $\mathrm{N}$ is the number of distances used, $d_{i}$ is the distance between two corresponding points and $\alpha$ is a parameter related to the size of the contours. The value of $\alpha=1 / 9$ was chosen so that, if all the distances are equal to 3 pixels, $F_{\text {Pratt }}$ will have the value 0.5 .

The mean distance was used to obtain a global average view of the distance between contours. The Pratt figure of merit also corresponds to a measure of the global behaviour of distances between contours; however it is a relative measure that varies in the interval $[0,1]$. The maximum distance allows assessing the worst-case values.

Finally, another possible way of comparing two contours consists in using the binary images defined by these contours and calculate their similarity. Two binary images are defined having the pixels interior to areas C and D set to " 1 ” and all the others set to " 0 ”. If these images have $n$ pixels, two $n$ dimensional vectors and $\vec{I}$ are defined having as components the values of the pixels ( 1 or 0$)$. These binary images may be regarded as elements of a Hilbert vector space where, using the usual definitions of norm and inner product, one can obtain a morphological similarity measure based on the parameter:

$$
\theta=\arccos \left(\frac{\vec{H} \cdot \vec{I}}{\|\vec{H}\|\|\vec{I}\|}\right)
$$

The similarity angle, $\theta$, measures the similarity between the two contours. This value will vary from a value of 0 degrees, for coincident contours, to 90 degrees for completely different contours.

\subsection{EXPERIMENTAL SETUP}

We have asked six experts, most of them having many years of experience, two from each of three different imagiology departments, to manually-draw 60 pulmonary contours on the 30 selected images. We have also detected pulmonary contours on the same images using our segmentation algorithm; thus we have used in the inter-observer variability study contours detected by seven detectors (six humans and one digital).

In order to study the intra-observer variability we have asked two experts of the same imagiology department to detect the contours on the same images a month later without telling them that they had already drawn contours on those 
images. These experts had a significantly different experience, one of them had many years of experience and the other had recently completed his training as an imagiologist.

\subsection{STATISTICAL METHODS}

The first step to process the data obtained from all the contours using the mentioned figures of merit, was to perform an Exploratory Data Analysis (EDA) ${ }^{14}$. The results have provided general information on the structural relations, showing the amplitudes, asymmetries, localizations, outliers, etc, and some clues to hypothesis formalization that could be tested. As the sample set did not correspond to independent experiments nor the data had a normal distribution, a nonparametric test was used ${ }^{15}$. We have also used Multivariate Data Analysis (MDA) ${ }^{16}$ to assess if our algorithm is generally comparable to the six human observers as a contour detector on the used image data set.

\section{RESULTS}

In this section we present the results obtained, using the described methods, in the studies performed on inter- and intra-observer variability. In most cases we show only results obtained using the Pratt figure of merit since we have concluded in a previous study ${ }^{9}$, and confirmed through this one, that all figures of merit except for the maximum distance produce consistent results, conveying the same type of information.

As a first approach we have studied the inter-observer variability among all experts and the algorithm in a worst-case scenario; this was performed using the reference contour and through the maximum error figure of merit and Exploratory Data Analysis (EDA). Then we have analysed the intra-observer variability comparing directly the contours drawn by two experts in two different moments and using the Pratt figure of merit and EDA. Finally, in order to assess if our algorithm is comparable as a contour detector to the six expert imagiologists, we have studied (in relation to the reference) the performance of all detectors (humans and algorithm) using several figures of merit, Exploratory and Multivariate Data Analysis.

Figure 11 shows the box plots of the data resulting from the comparison of the contours obtained by each detector (humans and algorithm) to the reference contours using the maximum error (distance). On these plots we observe a concentration of the smaller values, some outliers for all detectors (corresponding to images that should be analysed) and median values for all detectors between 5.4 and 9.9 pixels; these values that can be considered low for images of 512x512 pixels. Thus, even in this case all the detectors seem to have a good performance in the used image data set.

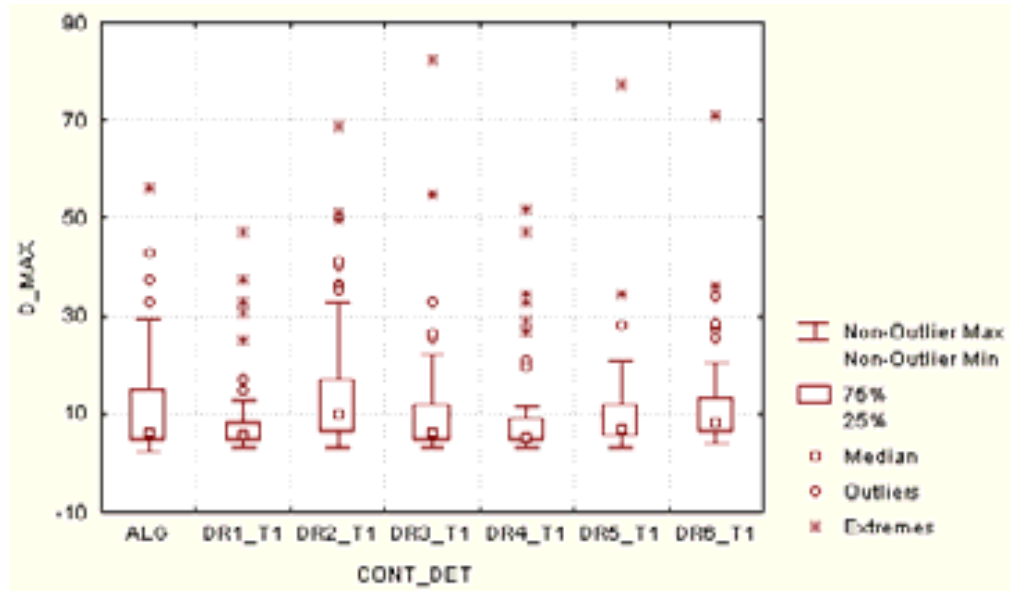

Figure 11 - Inter-observer variability: box plots corresponding to the comparison (to the reference) of the contours detected by each detector (DR1 to DR6 and the algorithm ALG ) using the maximum distance figure of merit.

To study the intra-observer variability of two doctors (DR1and DR2) we have compared directly the two contours they have drawn (in moments $\mathrm{T} 1$ and $\mathrm{T} 2$ ) on each image. Since both doctors were considered expert imagiologists, although they had a different experience, this study was expected to give us a measure of acceptable variability. Figure 12 shows 
the box plots corresponding to the Pratt figure of merit obtained for this comparison. We can see that the median values are 0.76 and 0.85 , respectively. A Wilcoxon matched pairs test ${ }^{15}$ rejected the equality of medians $(p<0,0002)$ for DR1_T1 and DR1_T2 as well as for DR2_T1 and DR2_T2. This confirms that the observed variability did not result from random causes.

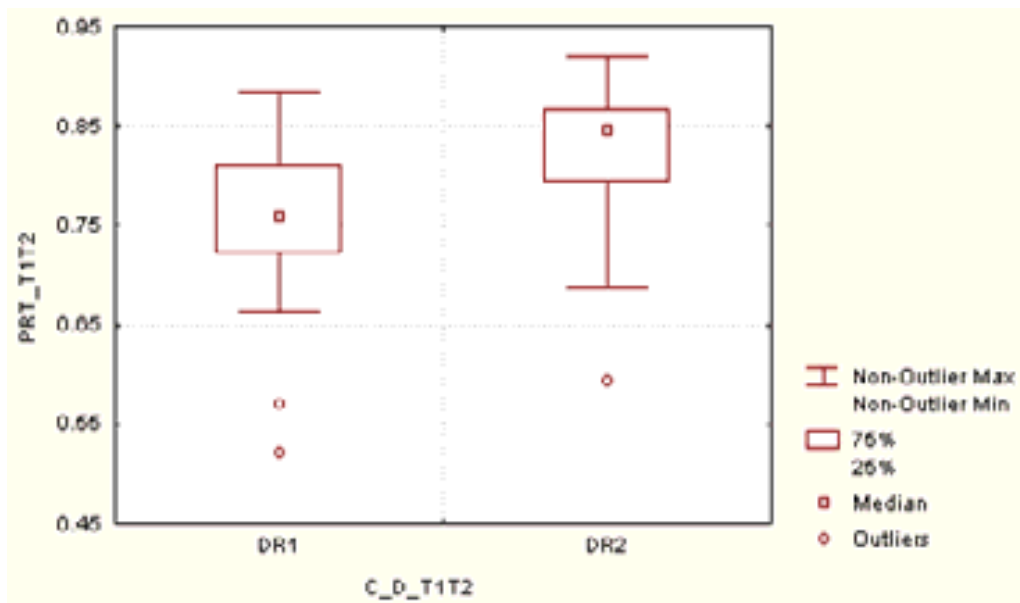

Figure 12 - Intra-observer variability using the Pratt figure of merit: box plots corresponding to the direct comparison of the contours detected by each doctor (DR1 and DR2) in two different moments (T1 and T2).

After this direct assess of the intra-observer variability we have compared the variability in relation to the reference contour. In this study we have included the contours drawn by all the doctors (DR1 to DR6) in the first time, the contours drawn by DR1 and DR2 in the second time (as DR1_T2 and DR2_T2), as well as the contours obtained using our algorithm (ALG). Observing figure 13, which shows the box plots obtained from these data using the Pratt figure of merit, we notice that the median value obtained for our algorithm is quite similar to the value for doctors DR4_T1, higher than the values for doctors DR1_T2, DR3_T1, DR5_T1, DR6_T1 and lower than the values for doctors DR1_T1, DR2_T1, DR2_T2. This seems to indicate that our algorithm produced, for the used image set, contours more similar to the reference than a significant part of the expert imagiologists.

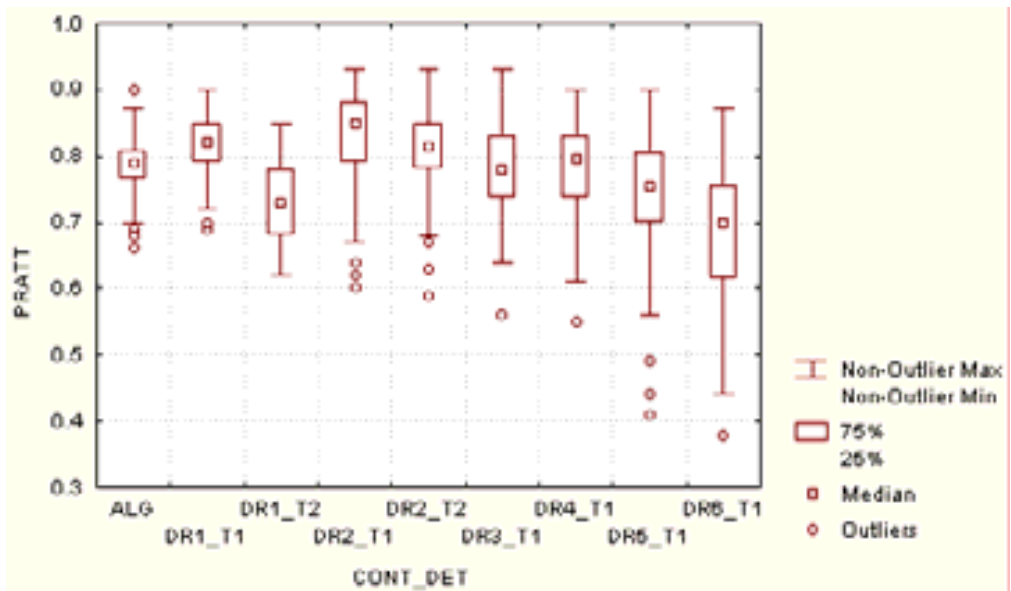

Figure 13 - Intra- and inter-observer variability using the Pratt figure of merit: box plots corresponding to the comparison to the reference of the contours detected by each doctor in the first time (DR1_T1 to DR6_T1), two doctors in the second time (DR1_T2 and DR2_T2) and the algorithm.

The above result suggested that we should explore further the relation among the performance of our algorithm as a detector to the performance of all the doctors. Thus we have used Clustering Analysis, which closely associated our algorithm with DR1_T1 as shown by the dendogram plot of figure 14; this means that, in this context, our algorithm is more similar to doctor DR1 than he is to himself in different moments, namely DR1_T1 and DR1_T2. 


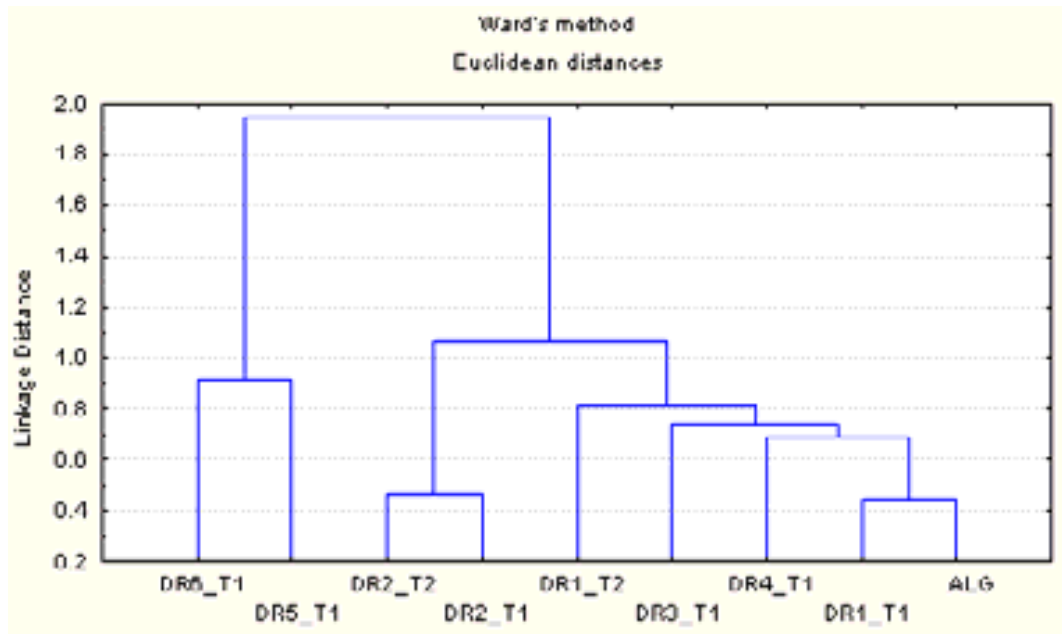

Figure 14 - Intra- and inter-observer variability using the Pratt figure of merit: dendogram plot (Clustering Analysis) showing all the doctors in the first time (DR1_T1 to DR6_T1), two doctors in the second time (DR1_T2 and DR2_T2) and the algorithm (ALG).

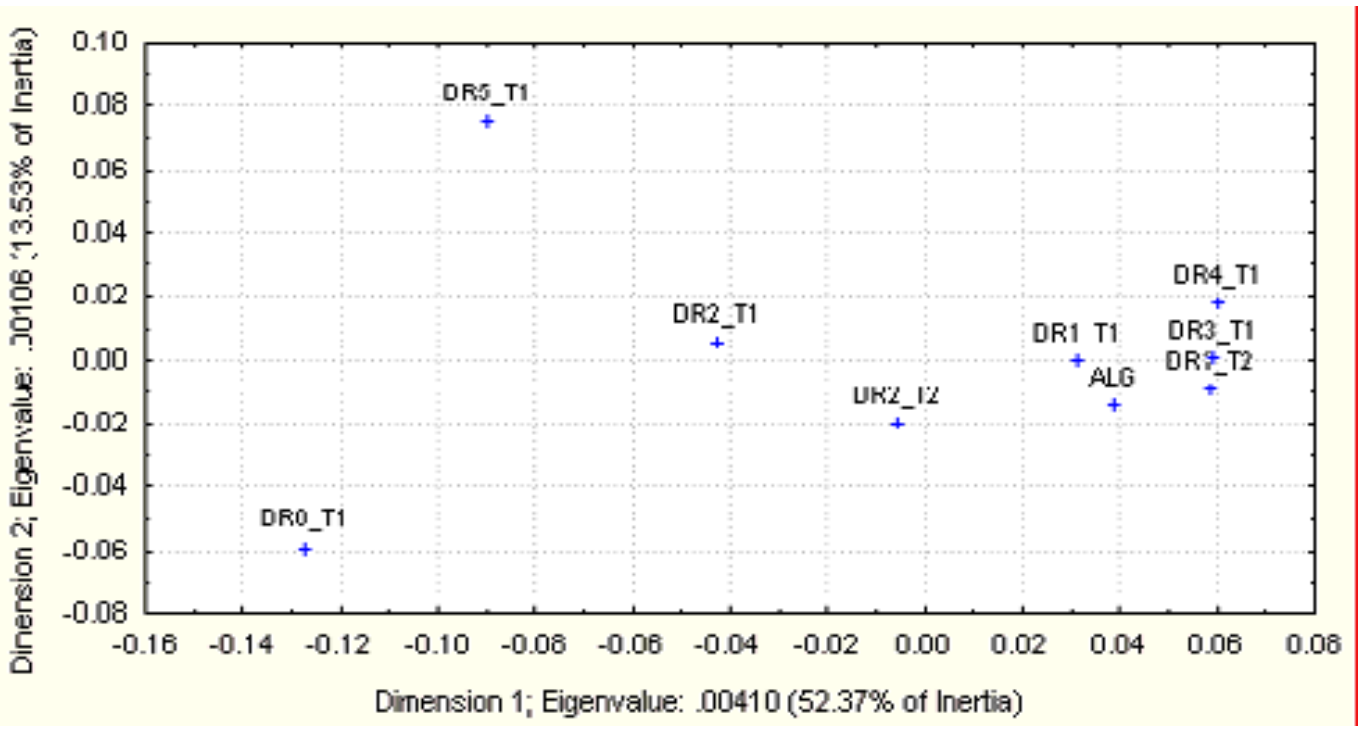

Figure 15 - Intra- and inter-observer variability using the Pratt figure of merit: Correspondence Analysis plot showing all the doctors in the first time (DR1_T1 to DR6_T1), two doctors in the second time (DR1_T2 and DR2_T2) and the algorithm (ALG).

A confirmation of this result was obtained through the use of another method of Multivariate Data Analysis. Figure 15 shows the projection on the plane defined by the first two axis ( $\approx 66 \%$ of the total inertia) of a Correspondence Analysis. Observing this figure we notice that our algorithm is clearly included in a group of four doctors (DR1_T1, DR1_T2, DR3_ T1, DR4_T1), doctors DR5 and DR6 seem to form another group and DR2 is isolated between the two groups. Note that the same conclusion could be drawn from the dendogram of figure 14. This could be related to the facts that doctors DR5 and DR6 work in the same department, (different from the others) and perhaps use different segmentation criteria, doctor DR2 has just finished his training as an imagiologist and all the others have a much larger experience.

In order to obtain a global average view of the distance between detected contours and the reference we have used the mean-distance figure of merit. Figure 16 shows the box plots for all the detectors (humans and algorithm); observing this figure we can notice that the median values are less than two pixels $(<1 \%)$, in all cases except for DR6, a good value considering the image resolution of 512x512. 


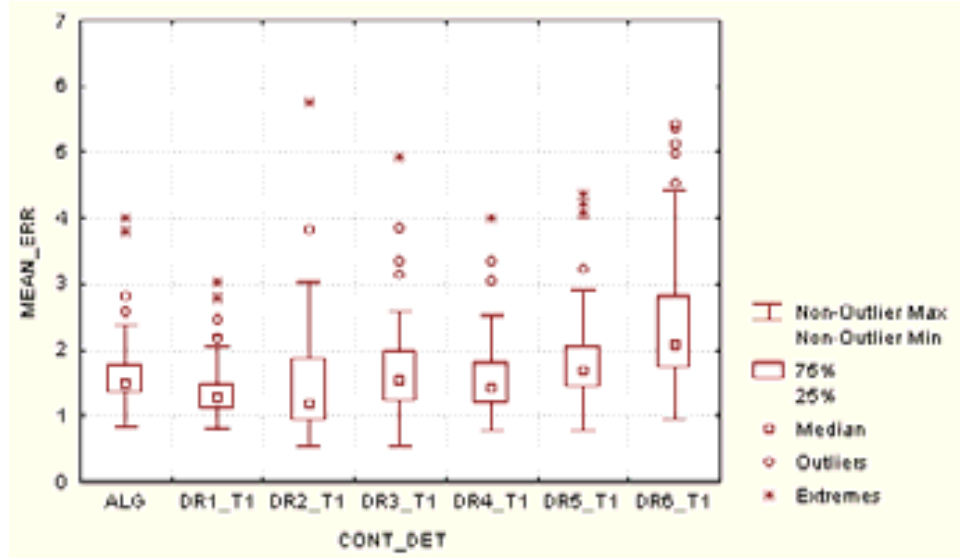

Figure 16 - Inter-observer variability using the mean distance: box plots corresponding to the comparison to the reference of the contours detected by each doctor in the first time (DR1_T1 to DR6_T1) and the algorithm (ALG).

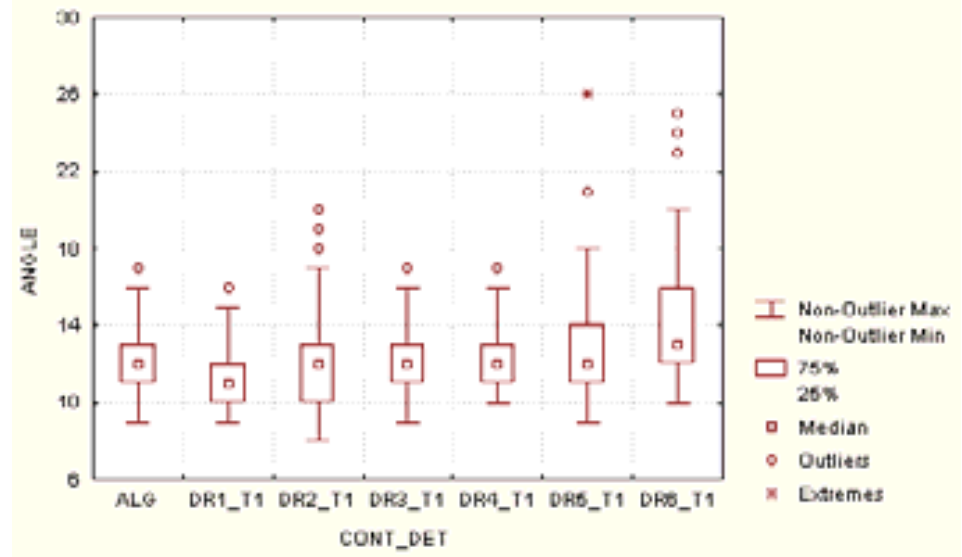

Figure 17 - Inter-observer variability using the similarity angle: box plots showing all the doctors in the first time (DR1_T1 to DR6_T1) and the algorithm (ALG).

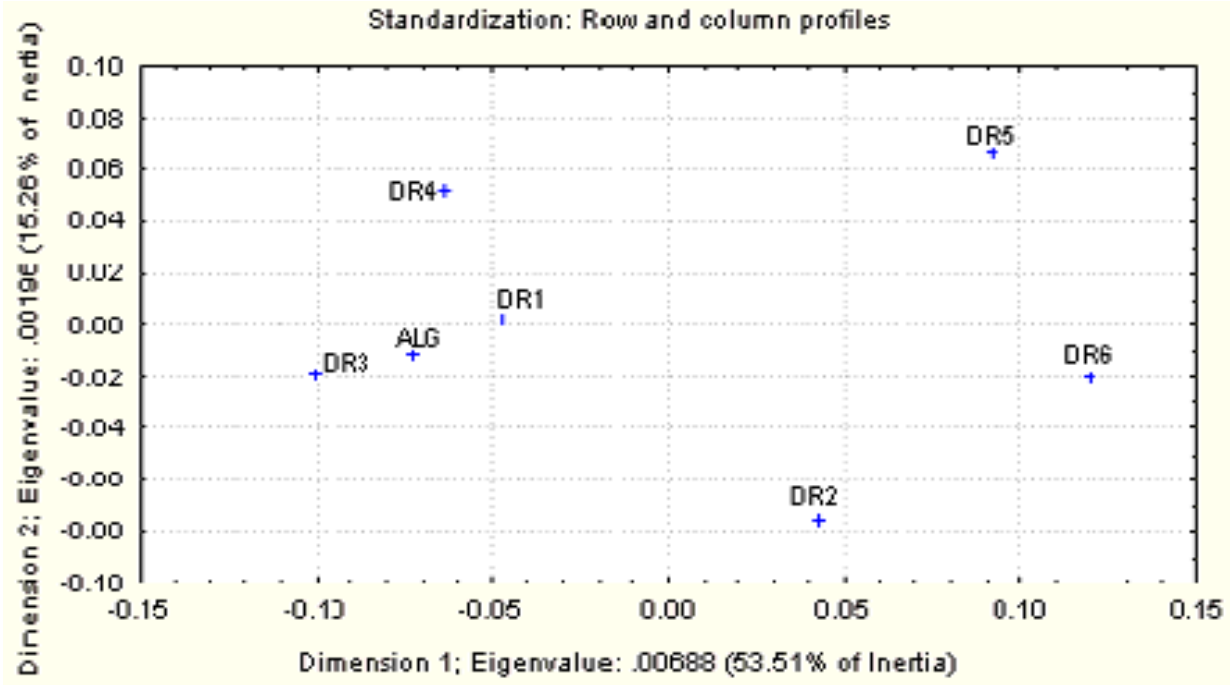

Figure 18 - Inter-observer variability using the similarity angle: Correspondence Analysis plot showing all the doctors in the first time (DR1_T1 to DR6_T1) and the algorithm (ALG). 
Finally, using the similarity angle we have obtained a confirmation of the results previously found through the Pratt figure of merit. On figure 17 we can notice that the box plots of all the doctors and the algorithm have a general relation among them inverse to the one observed in figure 13; for the similarity angle, the lower the value, the similar the contours are to the reference; inversely for the Pratt figure of merit the higher the value, the similar the contours are to reference. Moreover, the projection on the first two axis ( $\approx 69 \%$ of the total inertia) obtained through Correspondence Analysis (shown in figure 18) suggests the same grouping as obtained using the Pratt figure of merit: DR1_T1, DR3_T1, DR4_T1 and our algorithm seem to form one group, DR5 and DR6 another group and DR2 is isolated between the two groups.

\section{CONCLUSIONS}

In this work we have described a quantitative evaluation of the performance of a pulmonary segmentation algorithm based on the study of the inter-observer variability among six experts from different imagiology departments and the algorithm, as well as, the intra-observer variability of two experts from the same department having different experience. This study was performed comparing the contours detected by all experts and the algorithm to a reference contour obtained from the contours drawn by the six imagiologists. We have applied several figures of merit and a statistically supported methodology using Exploratory Data Analysis, Non Parametric Tests and Multivariate Analysis.

As an overall result of this comparison we are able to claim that the consistency and accuracy of our pulmonary segmentation algorithm must be adequate for the quantitative requirements mentioned by the imagiologists, in most cases. We base this assertion on the fact that we have been able to show, through different quantitative methods and for a data set of 60 contours, that the performance of our algorithm, as a contour detector, is similar to the performance of six expert imagiologists. Moreover we have shown that our algorithm was more similar to one of the experts than he was to himself in two different moments.

The used reference contour, which corresponds to a "mean" contour, seems acceptable in most images with diagnosis value. However, in a few images of the data set, the six imagiologists detected contours that seem to correspond to the use of different segmentation criteria in particular regions; in these situations a "mean" contour does not make sense as a reference and a different approach should be used. This needs to be further investigated.

The used methodology was developed specifically to quantitatively evaluate the performance of our pulmonary region segmentation algorithm; nevertheless we believe it is general enough to be applicable to many other problems of segmentation on medical images.

\section{ACKNOWLEDGEMENTS}

The authors are grateful to Drs. Luisa Teixeira and Agostinho from Hospitais da Universidade, Coimbra, to Drs. Jorge Pinho e Melo and Rui Pinho Melo from CENTAC, Aveiro and to Drs. Anabela Fidalgo and Fernando Figueiredo from Hospital Infante D. Pedro, Aveiro for manually detecting all contours.

\section{REFERENCES}

1. A. Hasegawa, S.-C. B. Lo, J.-S. Lin, M. T. Freedman, and S. K. Mun, "A Shift-invariant Neural Network for the Lung Field Segmentation in Chest Radiography", Journal of VLSI Signal Processing, vol. 18, pp. 241-250, 1998.

2. J. Brink and et al., "Helical CT: Principles and Technical Considerations", RadioGraphics, vol. 14, no. 4, pp. 887 893, 1994.

3. R. P. Parker, "Measurement of Basic CT Data", Proceedings of Physical Aspects of Medical Imaging, pp. 291-295, Manchester, U.K., 1980. 
4. A. Silva, J. S. Silva, B. S. Santos, and C. Ferreira, "Fast Pulmonary Contour Extraction in X-ray CT Images: A Methodology and Quality Assessment", SPIE - Medical Imaging 2001: Physiology and Function from Multidimensional Images, vol. 4321, pp. 216-224, 2001.

5. M. D. Heath, S. Sarkar, T. Sanocki, and K. W. Bowyer, "A Robust Visual Method for Assessing the Relative Performance of Edge-Detection Algorithms", IEEE Transactions on Pattern Analysis and Machine Intelligence, vol. 19, no. 12, pp. 1338-1359, 1997.

6. K. W. Bowyer, Validation of Medical Image Analysis Techniques (cap. XX), vol. 2: SPIE - The International Society for Optical Engineering, 1999.

7. V. Chalana and Y. Kim, "A Methodology for evaluation of Boundary Detection Algorithms on Medical Images", IEEE Transactions on Pattern Analysis and Machine Intelligence, vol. 16, no. 5, pp. 642-652, 1997.

8. B. Shahraray and D. J. Anderson, "Optimal Estimation of Contour Properties by Cross-Validated Regularization", IEEE Transactions on Pattern Analysis and Machine Intelligence, vol. 11, no. 6, pp. 600-610, 1989.

9. J. S. Silva, C. Ferreira, B. S. Santos, A. Silva, P. Agostinho, and L. Teixeira, "Comparing Inter- and Intra observer Variability of a Segmentation Algorithm and Expert Radiologists in Pulmonary Contours Extration", Abstracts of the 6th Portuguese Conference on Biomedical Engineering - BIOENG 2001, pp. 123-124 (6 pages extended version available in proceedings conference CD), Faro - Portugal, 2001.

10. I. Buvat and e. al., "The need to develop guidelines for the evaluation of medical image processing procedures", SPIE - Medical Imaging 1999: Image Processing, pp. 1466-1477, U.S.A., 1999.

11. S. R. Deans, Radon and Abel Transforms, Russ ed: CRC Press, 1996.

12. C. R. Giardina and E. R. Dougherty, Morphological Methods in Image and Signal Processing. New Jersey: Prentice Hall, 1988.

13. I. E. Abdou and W. K. Pratt, "Quantitative Design and Evaluation of Enhancement / Thresholding Edge Detectors", Proceedings of the IEEE, vol. 67, no. 5, pp. 753-763, 1979.

14. D. Hoaglin, F. Mosteller, and J. Tukey, Analise Exploratoria de Dados - Tecnicas Robustas: Um Guia: Salamandra, 1992.

15. D. G. Altman, Practical Statistics for Medical Research. Chapman \& Hall, 1999.

16. D. G. Johnson, Applied Multivariate Methods for Data Analysts. Duxbury Press, 1998. 